\title{
Thermodynamic formalism for some systems with countable Markov structures
}

\author{
Michael Jakobson \\ May 13, 2019 \\ To the memory of Dmitry Viktorovich Anosov

\begin{abstract}
We study ergodic properties of certain piecewise smooth two-dimensional systems by constructing countable Markov partitions. Using thermodynamic formalism we prove exponential decay of correleations. That extends the results of [8], [9]. Our approach is motivated by the original method of Anosov and Sinai from [5].
\end{abstract}

\section{Motivation: Folklore Theorem in dimension 1}

A well-known Folklore Theorem in one-dimensional dynamics can be formulated as follows.

Folklore Theorem. Let $I=[0,1]$ be the unit interval, and suppose $\left\{I_{1}, I_{2}, \ldots\right\}$ is a countable collection of disjoint open subintervals of I such that $\bigcup_{i} I_{i}$ has the full Lebesgue measure in I. Suppose there are constants $K_{0}>1$ and $K_{1}>0$ and mappings $f_{i}: I_{i} \rightarrow I$ satisfying the following conditions.

1. $f_{i}$ extends to a $C^{2}$ diffeomorphism from the closure of $I_{i}$ onto $[0,1]$, and $\inf _{z \in I_{i}}\left|D f_{i}(z)\right|>K_{0}$ for all $i$.

2. $\sup _{z \in I_{i}} \frac{\left|D^{2} f_{i}(z)\right|}{D f_{i}(z) \mid}\left|I_{i}\right|<K_{1}$ for all $i$. 
Then, the mapping $F(z)$ defined by $F(z)=f_{i}(z)$ for $z \in I_{i}$, has a unique invariant ergodic probability measure $\mu$ equivalent to Lebesgue measure on $I$.

For the proof of the Folklore theorem, the ergodic properties of $\mu$ and the history of the question see for example [3] and [15].

In [8], [9] the Folklore Theorem was generalized to two-dimensional maps $F$ which piecewise coincide with certain hyperbolic diffeomorphisms $f_{i}$. As in the one-dimensional situation there is an essential difference between a finite and an infinite number of $f_{i}$. In the case of an infinite number of $f_{i}$, their derivatives grow with $i$ and relations between first and second derivatives become crucial.

Models with infinitely many $f_{i}$ appear when we study non-hyperbolic systems, such as quadratic-like maps in dimension 1, and Henon-like maps in dimension 2.

\section{Model under consideration. Geometric and hy- perbolicity conditions}

1. As in [8], [9] we consider the following 2-d model. Let $Q$ be the unit square. Let $\xi=\left\{E_{1}, E_{2}, \ldots,\right\}$ be a countable collection of closed curvilinear rectangles in $Q$. Assume that each $E_{i}$ lies inside a domain of definition of a $C^{2}$ diffeomorphism $f_{i}$ which maps $E_{i}$ onto its image $S_{i} \subset Q$. We assume each $E_{i}$ connects the top and the bottom of $Q$. Thus each $E_{i}$ is bounded from above and from below by two subintervals of the line segments $\{(x, y): y=1,0 \leq x \leq 1\}$ and $\{(x, y): y=0,0 \leq x \leq 1\}$. Hyperbolicity conditions that we formulate below imply that the left and right boundaries of $E_{i}$ are graphs of smooth functions $x^{(i)}(y)$ with $\left|\frac{d x^{(i)}}{d y}\right| \leq \alpha$ where $\alpha$ is a real number satisfying $0<\alpha<1$.

The images $f_{i}\left(E_{i}\right)=S_{i}$ are narrow strips connecting the left and right sides of $Q$ and that they are bounded on the left and right by the two subintervals of the line segments $\{(x, y): x=0,0 \leq y \leq 1\}$ and $\{(x, y): x=1,0 \leq y \leq 1\}$ and above and below by the graphs of smooth functions $Y^{i}(X),\left|\frac{d Y^{(i)}}{d X}\right| \leq \alpha$. We are saying that $E_{i}^{\prime}$ s are full height in $Q$ while the $S_{i}^{\prime} s$ are full width in $Q$.

2. For $z \in Q$, let $\ell_{z}$ be the horizontal line through $z$. We define $\delta_{z}\left(E_{i}\right)=$ $\operatorname{diam}\left(\ell_{z} \cap E_{i}\right), \delta_{i, \max }=\max _{z \in Q} \delta_{z}\left(E_{i}\right), \delta_{i, \min }=\min _{z \in Q} \delta_{z}\left(E_{i}\right)$. We assume the following 


\section{Geometric conditions.}

G1. For $i \neq j$ holds int $E_{i} \cap$ int $E_{j}=\emptyset$ int $S_{i} \cap$ int $S_{j}=\emptyset$.

G2. mes $\left(Q \backslash \cup_{i}\right.$ int $\left.E_{i}\right)=0$ where mes stands for Lebesgue measure.

G3. $-\sum_{i} \delta_{i, \max } \log \delta_{i, \min }<\infty$.

3. In the standard coordinate system for a map $F:(x, y) \rightarrow\left(F_{1}(x, y), F_{2}(x, y)\right)$ we use $D F(x, y)$ to denote the differential of $F$ at some point $(x, y)$ and $F_{j x}$, $F_{j y}, F_{j x x}, F_{j x y}$, etc., for partial derivatives of $F_{j}, j=1,2$.

Let $J_{F}(z)=\left|F_{1 x}(z) F_{2 y}(z)-F_{1 y}(z) F_{2 x}(z)\right|$ be the absolute value of the Jacobian determinant of $F$ at $z$.

\section{Hyperbolicity conditions.}

There exist constants $0<\alpha<1$ and $K_{0}>1$ such that for each $i$ the map

$$
F(z)=f_{i}(z) \text { for } z \in E_{i}
$$

satisfies

H1. $\left|F_{2 x}(z)\right|+\alpha\left|F_{2 y}(z)\right|+\alpha^{2}\left|F_{1 y}(z)\right| \leq \alpha\left|F_{1 x}(z)\right|$

H2. $\left|F_{1 x}(z)\right|-\alpha\left|F_{1 y}(z)\right| \geq K_{0}$.

H3. $\left|F_{1 y}(z)\right|+\alpha\left|F_{2 y}(z)\right|+\alpha^{2}\left|F_{2 x}(z)\right| \leq \alpha\left|F_{1 x}(z)\right|$

H4. $\left|F_{1 x}(z)\right|-\alpha\left|F_{2 x}(z)\right| \geq J_{F}(z) K_{0}$.

For a real number $0<\alpha<1$, we define the cones

$$
\begin{aligned}
& K_{\alpha}^{u}=\left\{\left(v_{1}, v_{2}\right):\left|v_{2}\right| \leq \alpha\left|v_{1}\right|\right\} \\
& K_{\alpha}^{s}=\left\{\left(v_{1}, v_{2}\right):\left|v_{1}\right| \leq \alpha\left|v_{2}\right|\right\}
\end{aligned}
$$

and the corresponding cone fields $K_{\alpha}^{u}(z), K_{\alpha}^{s}(z)$ in the tangent spaces at points $z \in \mathbf{R}^{2}$.

The following proposition proved in [9] relates conditions H1-H4 above with the usual definition of hyperbolicity in terms of cone conditions. It shows that conditions $\mathrm{H} 1$ and $\mathrm{H} 2$ imply that the $K_{\alpha}^{u}$ cone is mapped into 
itself by $D F$ and expanded by a factor no smaller than $K_{0}$ while $\mathrm{H} 3$ and $\mathrm{H} 4$ imply that the $K_{\alpha}^{s}$ cone is mapped into itself by $D F^{-1}$ and expanded by a factor no smaller than $K_{0}$.

Unless otherwise stated, we use the $\max$ norm on $\mathbf{R}^{2},\left|\left(v_{1}, v_{2}\right)\right|=\max \left(\left|v_{1}\right|,\left|v_{2}\right|\right)$.

Proposition 2.1 Under conditions H1-H4 above, we have

$$
\begin{gathered}
D F\left(K_{\alpha}^{u}\right) \subseteq K_{\alpha}^{u} \\
v \in K_{\alpha}^{u} \Rightarrow|D F v| \geq K_{0}|v| \\
D F^{-1}\left(K_{\alpha}^{s}\right) \subseteq K_{\alpha}^{s} \\
v \in K_{\alpha}^{s} \Rightarrow\left|D F^{-1} v\right| \geq K_{0}|v|
\end{gathered}
$$

Remark 2.2 The first version of hyperbolicity conditions appeared in [14]. It was developed in particular in [4] and [7] . Here we use hyperbolicity conditions from [9]. In [8] we used hyperolicity conditions from [4] which implied the invariance of cones and uniform expansion with respect to the sum norm $|v|=\left|v_{1}\right|+\left|v_{2}\right|$.

4. The map

$$
F(z)=f_{i}(z) \text { for } z \in \text { int } E_{i}
$$

is defined almost everywhere on $Q$. Let $\tilde{Q}_{0}=\bigcup_{i}$ int $E_{i}$, and, define $\tilde{Q}_{n}, n>0$, inductively by $\tilde{Q}_{n}=\tilde{Q}_{0} \bigcap F^{-1} \tilde{Q}_{n-1}$. Let $\tilde{Q}=\bigcap_{n \geq 0} \tilde{Q}_{n}$ be the set of points whose forward orbits always stay in $\bigcup_{i}$ int $E_{i}$. Then, $\tilde{Q}$ has full Lebesgue measure in $Q$, and $F$ maps $\tilde{Q}$ into itself.

The hyperbolicity conditions $\mathrm{H} 1-\mathrm{H} 4$ imply the estimates on the derivatives of the boundary curves of $E_{i}$ and $S_{i}$ which we described earlier. They also imply that any intersection $f_{i} E_{i} \cap E_{j}$ is full width in $E_{j}$. Further, $E_{i j}=$ 
$E_{i} \cap f_{i}^{-1} E_{j}$ is a full height subrectangle of $E_{i}$ and $S_{i j}=f_{j} f_{i} E_{i j}$ is a full width substrip in $Q$.

Given a finite string $i_{0} \ldots i_{n-1}$, we define inductively

$$
E_{i_{0} \ldots i_{n-1}}=E_{i_{0}} \bigcap f_{i_{0}}^{-1} E_{i_{1} i_{2} \ldots i_{n-1}} .
$$

Then, each set $E_{i_{0} \ldots i_{n-1}}$ is a full height subrectangle of $E_{i_{0}}$.

Analogously, for a string $i_{-m} \ldots i_{-1}$ we define

$$
S_{i_{-m} \ldots i_{-1}}=f_{i_{-1}}\left(S_{i_{-m} \ldots i_{-2}} \bigcap E_{i_{-1}}\right)
$$

and get that $S_{i_{-m} \ldots i_{-1}}$ is a full width strip in $Q$. It is easy to see that $S_{i_{-m} \ldots i_{-1}}=$ $f_{i_{-1}} \circ f_{i_{-2}} \circ \ldots \circ f_{i_{-m}}\left(E_{i_{-m} \ldots i_{-1}}\right)$ and that $f_{i_{0}}^{-1}\left(S_{i_{-m} \ldots i_{-1}}\right)$ is a full-width substrip of $E_{i_{0}}$.

We also define curvilinear rectangles $R_{i_{-m} \ldots i_{-1}, i_{0} \ldots i_{n-1}}$ by

$$
R_{i_{-m} \ldots i_{-1}, i_{0} \ldots i_{n-1}}=S_{i_{-m} \ldots i_{-1}} \bigcap E_{i_{0} \ldots i_{n-1}}
$$

If there are no negative indices then respective rectangle is full height in $Q$. For infinite strings, we have the following Proposition.

Proposition 2.3 Any $C^{1}$ map $F$ satisfying the above geometric conditions G1-G3 and hyperbolicity conditions H1-H4 has a "topological attractor"

$$
\Lambda=\bigcup_{\ldots i_{-n} \ldots i_{-1}} \bigcap_{k \geq 1} S_{i_{-k} \ldots i_{-1}}
$$

The infinite intersections $\bigcap_{k=1}^{\infty} S_{i_{-k} \ldots i_{-1}}$ define $C^{1}$ curves $y(x),|d y / d x| \leq \alpha$ which are the unstable manifolds for the points of the attractor. The infinite intersections $\bigcap_{k=1}^{\infty} E_{i_{0} \ldots i_{k-1}}$ define $C^{1}$ curves $x(y),|d x / d y| \leq \alpha$ which are the stable manifolds for the points of the attractor. The infinite intersections

$$
\bigcap_{m=1}^{\infty} \bigcap_{n=1}^{\infty} R_{i_{-m} \ldots i_{-1}, i_{0} \ldots i_{n-1}}
$$

define points of the attractor. 
Proposition 2.3 is a well known fact in hyperbolic theory. For example it follows from Theorem 1 in [4]. See also [10]. The union of the stable manifolds has full measure in $Q$. The trajectories of all points in this set converge to $\Lambda$. That is the reason to call $\Lambda$ a topological attractor.

5. An $F$-invariant Borel probablility measure $\mu$ on $Q$ is called a SinaiRuelle-Bowen measure (or SRB-measure) for $F$ if $\mu$ is ergodic and there is a set $A \subset Q$ of positive Lebesgue measure such that for $x \in A$ and any continuous real-valued function $\phi: Q \rightarrow \mathbf{R}$, we have

$$
\lim _{n \rightarrow \infty} \frac{1}{n} \sum_{k=0}^{n-1} \phi\left(F^{k} x\right)=\int \phi d \mu .
$$

Existence of an SRB measure is a much stronger result, than 2.3. It allows to describe statistical properties of trajectories in a set of positive phase volume. It requires some additional assumptions.

\section{Distortion conditions}

As we have a countable number of domains the derivatives of $f_{i}$ grow. We formulate certain assumptions on the second derivatives. We use the distance function $d\left((x, y),\left(x_{1}, y_{1}\right)\right)=\max \left(\left|x-x_{1}\right|,\left|y-y_{1}\right|\right)$ associated with the norm $|v|=$ $\max \left(\left|v_{1}\right|,\left|v_{2}\right|\right)$ on vectors $v=\left(v_{1}, v_{2}\right)$.

As above, for a point $z \in Q$, let $l_{z}$ denote the horizontal line through $z$, and if $E \subseteq Q$, let $\delta_{z}(E)$ denote the diameter of the horizontal section $l_{z} \cap E$. We call $\delta_{z}(E)$ the $z-$ width of $E$.

In given coordinate systems we write $f_{i}(x, y)=\left(f_{i 1}(x, y), f_{i 2}(x, y)\right)$. We use $f_{i j x}, f_{i j y}, f_{i j x x}, f_{i j x y}$, etc. for partial derivatives of $f_{i j}, j=1,2$.

We define

$$
\left|D^{2} f_{i}(z)\right|=\max _{j=1,2,(k, l)=(x, x),(x, y),(y, y)}\left|f_{i j k l}(z)\right|
$$

Next we formulate distortion conditions which are used to control the fluctuation of the derivatives of iterates of $F$ along unstable manifolds, and to construct Sinai local measures.

Suppose there is a constant $C_{0}>0$ such that the following distortion conditions hold 
D1. $\sup _{z \in E_{i}, i \geq 1} \frac{\left|D^{2} f_{i}(z)\right|}{\left|f_{i 1 x}(z)\right|} \delta_{z}\left(E_{i}\right)<C_{0}$

Our conditions imply the following theorem proved in [8], [9].

Theorem 3.1 Let $F$ be a piecewise smooth mapping as above satisfying the geometric conditions $\mathrm{Gl}-\mathrm{G} 3$, the hyperbolicity conditions $\mathrm{HI}-\mathrm{H} 4$ and the distortion condition D1.

Then, $F$ has an SRB measure $\mu$ supported on $\Lambda$ whose basin has full Lebesgue measure in $Q$. Dynamical system $(F, \mu)$ satisfies the following properties.

1. $(F, \mu)$ is measure-theoretically isomorphic to a Bernoulli shift.

2. F has finite entropy with respect to the measure $\mu$, and the entropy formula holds

$$
h_{\mu}(F)=\int \log \left|D^{u} F\right| d \mu
$$

where $D^{u} F(z)$ is the norm of the derivative of $F$ in the unstable direction at $z$.

3.

$$
h_{\mu}(F)=\lim _{n \rightarrow \infty} \frac{1}{n} \log \left|D F^{n}(z)\right|
$$

where the latter limit exists for Lebesgue almost all $z$ and is independent of such $z$.

\section{Additional hyperbolicity and distortion conditions and statement of the main theorem}

When applying thermodynamic formalism to hyperbolic attractors one considers the function $\phi(z)=-\log \left(D^{u} F(z)\right)$. Thermodynamic formalism is based on the fact that the pullback of $\phi(z)$ into a symbolic space determined by some Markov partition is a locally Hölder function.

We prove Hölder property of $\phi(z)$ assuming an extra hyperbolicity condition, and a distortion condition D2 stronger than D1.

Hyperbolicity condition $\mathrm{H5}$. 
H5. $\frac{1}{K_{0}^{2}}+\alpha^{2}<1$

\section{Distortion condition D2.}

D2. $\sup _{z \in E_{i}, i \geq 1} \frac{\left|D^{2} f_{i}(z)\right|}{\left|f_{i 1 x}(z)\right|}<C_{0}$.

Remark 4.1 Condition D2 is too strong to be useful for quadratic-like systems. In dimension 1 it reads as $\left|\frac{F_{i x x}}{F_{i x}}\right|<c$ instead of $\left|\frac{F_{i x x}}{F_{i x}^{2}}\right|<c$. However instead of D2 one can assume additional hyperbolicity conditions, which can be vaguely formulated as "contraction of $f_{i}$ grows faster than expansion". That approach will be discussed in a forthcoming paper.

Assuming additionally H5 and D2 we prove that Hölder functions have exponential decay of correlations.

Let $\mathscr{H}_{\gamma}$ be the space of functions on $Q$ satisfying Hölder property with exponent $\gamma$

$$
|\phi(x)-\phi(y)| \leq c|x-y|^{\gamma}
$$

Then the following theorem holds.

Theorem 4.2 Let $F$ be a piecewise smooth mapping as above satisfying the geometric conditions G1-G3, the hyperbolicity conditions $\mathrm{H1}-\mathrm{H} 5$ and the distortion condition D2. Then $(F, \mu)$ has exponential decay of correlations for $\phi, \psi \in \mathscr{H}_{\gamma}$. Namely there exist $\eta(\gamma)<1$ and $C=C(\phi, \psi)$ such that

$$
\left|\int \phi\left(\psi \circ F^{n}\right) d \mu-\int \phi d \mu \int \psi d \mu\right|<C \eta^{n}
$$

\section{$5 \quad$ Hölder properties of $\log \left(D^{u} F(z)\right)$}

1. Although Markov partitions are partitions of the attractor, we need to check Hölder property on actual two-dimensional curvilinear rectangles $R_{i_{-m} \ldots i_{-1}, i_{0} \ldots i_{n-1}}$. We call respective partition Markov as well. In our model Markov partition consists of initial full height rectangles $E_{i}$.

We consider rectangles $R_{i_{-m} \ldots i_{-1}, i_{0} \ldots i_{n-1}}$ with $m \geq 0, n \geq 1$. We use notation 
$m=0$ if there are no negative coordinates, which means $R_{i_{-m} \ldots i_{-1}, i_{0} \ldots i_{n-1}}=$ $R_{i_{0}, \ldots, i_{n-1}}$ is a full height rectangle. The assumption $n \geq 1$, means that variations are measured between points which belong to the same full height rectangle.

By definition the function $\log D^{u} F$ is locally Hölder if for $m \geq 0, n \geq 1$ the variation of $\log D^{u} F$ on $R_{i_{-m} \ldots i_{-1}, i_{0} \ldots i_{n-1}}$ satisfies

$$
\operatorname{var}\left(\log D^{u} F\right) \mid R_{i_{-m} \ldots i_{-1}, i_{0} \ldots i_{n-1}}<C \theta_{0}^{\min (m, n)}
$$

for some $C>0, \theta_{0}<1$.

Proposition 5.1 $\log D^{u} F$ is a locally Hölder function.

We prove Proposition 5.1 with some $\theta_{0}$ and $C$ determined by hyperbolicity and distortion conditions.

(a) The sets $R_{i_{-m} \ldots i_{-1}, i_{0} \ldots i_{n-1}}$ are bounded from above and below by some arcs of two unstable curves $\Gamma_{i_{-m} \ldots i_{-1}}^{u}$, which are images of some pieces of the top and bottom of $\tilde{Q}$, and from left and right by some arcs of two stable curves $\Gamma_{i_{0} \ldots i_{n-1}}^{s}$, which are preimages of some pieces the left and right boundaries of $\tilde{Q}$.

Let $Z_{1}, Z_{2} \in R_{i_{-m} \ldots i_{-1}, i_{0} \ldots i_{n-1}}$ be two points on the attractor. We connect $Z_{1}, Z_{2}$ by two pieces of their unstable manifolds to two points $Z_{3}, Z_{4}$ which belong to the same stable manifold. Let

$\gamma_{1}=\gamma\left(Z_{1}, Z_{3}\right) \subset W^{u}\left(Z_{1}\right), \gamma_{2}=\gamma\left(Z_{2}, Z_{4}\right) \subset W^{u}\left(Z_{2}\right), \gamma_{3}=\gamma\left(Z_{3}, Z_{4}\right) \subset$ $W^{s}\left(Z_{3}\right)$ be respective curves all located inside $R_{i_{-m} \ldots i_{-1}, i_{0} \ldots i_{n-1}}$.

We estimate

$$
\begin{array}{r}
\left|\log D^{u} F\left(Z_{1}\right)-\log D^{u} F\left(Z_{2}\right)\right| \leq\left|\log D^{u} F\left(Z_{1}\right)-\log D^{u} F\left(Z_{3}\right)\right|+ \\
\quad\left|\log D^{u} F\left(Z_{3}\right)-\log D^{u} F\left(Z_{4}\right)\right|+\left|\log D^{u} F\left(Z_{4}\right)-\log D^{u} F\left(Z_{2}\right)\right|
\end{array}
$$

(b) First we estimate $\left|\log D^{u} F\left(Z_{1}\right)-\log D^{u} F\left(Z_{3}\right)\right|$. We connect $Z_{1}$ and $Z_{3}$ by a chain of small rectangles $R \subset R_{i_{-m} \ldots i_{-1}, i_{0} \ldots i_{n-1}}$ covering $\gamma_{1}$. Then $\left|\log D^{u} F\left(Z_{1}\right)-\log D^{u} F\left(Z_{3}\right)\right|$ is majorated by the sum of similar differences for points $z_{1}, z_{2} \in W^{u}\left(Z_{1}\right) \subset R$.

Because of cone conditions we can choose rectangles $R=\Delta x \times \Delta y$ satisfying $|\Delta y|<\alpha|\Delta x|$. Let $R$ be one of such rectangles.

Hyperbolicity conditions imply the following properties, see [9]. 
i. Any unit vector in $K_{\alpha}^{u}$ at a point $z \in E_{i}$, in particular a tangent vector to $W^{u}(z)$, has coordinates $\left(1, a_{z}\right)$ with $\left|a_{z}\right|<\alpha$.

ii.

$$
\left|D^{u} F(z)\right|=\left|F_{1 x}(z)+a_{z} F_{1 y}(z)\right|
$$

iii.

$$
\frac{\left|F_{1 y}\right|}{\left|F_{1 x}\right|}<\alpha
$$

iv.

$$
\frac{\left|F_{2 x}\right|}{\left|F_{1 x}\right|}<\alpha
$$

V.

$$
\frac{\left|F_{2 y}\right|}{\left|F_{1 x}\right|}<\frac{1}{K_{0}^{2}}+\alpha^{2}
$$

Assuming without loss of generality $F_{1 x}>0$ for all $x \in E_{i}$ we get that variation of $\log \left|D^{u} F\right|$ between two points $z_{1}, z_{2} \in W^{u}\left(Z_{1}\right) \subset R$ equals

$$
\log \left[F_{1 x}\left(z_{1}\right)\left(1+a_{z_{1}} \frac{F_{1 y}}{F_{1 x}}\left(z_{1}\right)\right)\right]-\log \left[F_{1 x}\left(z_{2}\right)\left(1+a_{z_{2}} \frac{F_{1 y}}{F_{1 x}}\left(z_{2}\right)\right)\right]
$$

We split it into two expressions and estimate separately

$$
\log F_{1 x}\left(z_{1}\right)-\log F_{1 x}\left(z_{2}\right)
$$

and

$$
\log \left(1+a_{z_{1}} \frac{F_{1 y}}{F_{1 x}}\left(z_{1}\right)\right)-\log \left(1+a_{z_{2}} \frac{F_{1 y}}{F_{1 x}}\left(z_{2}\right)\right)
$$

We rewrite 16 as

$$
\log \left(1+\frac{a_{z_{1}} \frac{F_{1 y}}{F_{1 x}}\left(z_{1}\right)-a_{z_{2}} \frac{F_{1 y}}{F_{1 x}}\left(z_{2}\right)}{1+a_{z_{2}} \frac{F_{1 y}}{F_{1 x}}\left(z_{2}\right)}\right)
$$

As denominator of the fraction in 17 is uniformly bounded away from 0 , we stimate the numerator and rewrite it as a sum of two expressions

$$
\left|a_{z_{1}}\right| \frac{\left|F_{1 y}\left(z_{1}\right) F_{1 x}\left(z_{2}\right)-F_{1 y}\left(z_{2}\right) F_{1 x}\left(z_{1}\right)\right|}{F_{1 x}\left(z_{1}\right) F_{1 x}\left(z_{2}\right)}
$$

and

$$
\left|a_{z_{1}}-a_{z_{2}}\right|\left|\frac{F_{1 y}\left(z_{2}\right)}{F_{1 x}\left(z_{2}\right)}\right|
$$


As $C^{2}$ sizes of unstable manifolds are uniformly bounded ( see [9]), 19 is bounded by $c|\Delta x|$. We rewrite 18 as

$$
a_{z_{1}}\left[\frac{F_{1 y}\left(z_{1}\right)\left(F_{1 x}\left(z_{2}\right)-F_{1 x}\left(z_{1}\right)\right)}{F_{1 x}\left(z_{1}\right) F_{1 x}\left(z_{2}\right)}+\frac{F_{1 y}\left(z_{1}\right)-F_{1 y}\left(z_{2}\right)}{F_{1 x}\left(z_{2}\right)}\right]
$$

As $\left|\frac{F_{1 y}\left(z_{1}\right)}{F_{1 x}\left(z_{1}\right)}\right|<\alpha$, both expressions are estimated similarly.

As we are moving along $W^{u}$, we get $|\Delta y|<\alpha|\Delta x|$.

We use the mean value theorem and distortion assumptions, and get estimates bounded by

$$
c|\Delta x| \frac{F_{1 x}(\theta)}{F_{1 x}\left(z_{2}\right)}
$$

Then it remains to estimate $\frac{F_{1 x}(\theta)}{F_{1 x}\left(z_{2}\right)}$ or equivalently $\left|\log F_{1 x}(\theta)-\log F_{1 x}\left(z_{2}\right)\right|$, which is the same estimate as 15 .

In order to estimate 15 we use again the mean value theorem and distortion assumptions.

Then we get

$$
\left|\log F_{1 x}(\theta)-\log F_{1 x}\left(z_{2}\right)\right|<c|\Delta x|
$$

and respectively

$$
\frac{F_{1 x}(\theta)}{F_{1 x}\left(z_{2}\right)}<\exp (c|\Delta x|)
$$

We combine the previous estimates and get

$$
\left|\log D^{u} F\left(z_{1}\right)-\log D^{u} F\left(z_{3}\right)\right|<c\left|\gamma\left(z_{1}, z_{3}\right)\right|
$$

From hyperbolicity conditions we get

$$
\left|\gamma\left(z_{1}, z_{3}\right)\right|<C_{2} \frac{1}{K_{0}^{n}}
$$

That implies

$$
\left|\log D^{u} F\left(z_{1}\right)-\log D^{u} F\left(z_{3}\right)\right|<C_{2} \frac{1}{K_{0}^{n}}
$$

where $C_{2}$ is a uniform constant.

Similar inequality holds for $\gamma\left(z_{2}, z_{4}\right)$.

$$
\left|\log D^{u} F\left(z_{2}\right)-\log D^{u} F\left(z_{4}\right)\right|<C_{2} \frac{1}{K_{0}^{n}}
$$


(c) Next we estimate the variation of $\log \left|D^{u} F(z)\right|$ between points $Z_{3}$ and $Z_{4}$, which belong to the same stable manifold $W^{s}\left(Z_{3}\right)=W^{s}\left(Z_{4}\right) \subset$ $R_{i_{-m} \ldots i_{-1}, i_{0} \ldots i_{n-1}}$. Thus we need to estimate

$$
\log \left|F_{1 x}\left(Z_{3}\right)+a_{Z_{3}} F_{1 y}\left(Z_{3}\right)\right|-\log \left|F_{1 x}\left(Z_{4}\right)+a_{Z_{4}} F_{1 y}\left(Z_{4}\right)\right|
$$

As above we split the variation 28 into 15,18 and 19 . This time instead of moving along $W^{u}\left(Z_{1}\right)$ we are moving along $W^{s}\left(Z_{3}\right)$, which connects $Z_{3}$ and $Z_{4}$. In that case we use $|\Delta x|<\alpha|\Delta y|$, so $\Delta y$ variations are added. As above estimates 15, 18 contribute less than

$$
c\left|\gamma_{3}\right|<C_{2} \frac{1}{K_{0}^{m}}
$$

Note that the Lipschitz dependence of the unstable leaves from $y \in W_{0}^{s}$ is unclear in our setting, differently from the classical case in dimension two.

The following lemma related to that issue is sufficient for our purposes.

Lemma 5.2 There exist $c_{0}>0,0<\theta_{0}<1$ such that

$$
\left|a_{Z_{3}}-a_{Z_{4}}\right|<c_{0} \theta_{0}^{m}
$$

Proof.

We assume by induction that for any rectangle $R_{i_{-m} \ldots i_{-1}, i_{0} \ldots i_{n-1}}$, and for any points $Z_{3}, Z_{4} \in R_{i_{-m} \ldots i_{-1}, i_{0} \ldots i_{n-1}}$ of intersection of two unstable manifolds $W_{1}^{u}, W_{2}^{u}$ with the same stable manifold $W_{0}^{s}$, the inequality 30 holds. Then we prove

$$
\left|a_{F\left(Z_{3}\right)}-a_{F\left(Z_{4}\right)}\right|<c_{0} \theta_{0}^{m+1}
$$

$D F$ maps a unit vector $\vec{v}=(1, a)$ into $\left(F_{1 x}+F_{1 y} a, F_{2 x}+F_{2 y} a\right)$. Then the normalized vector $D F \vec{v}$ has second coordinate

$$
a^{\prime}=\frac{\frac{F_{2 x}}{F_{1 x}}+\frac{F_{2 y}}{F_{1 x}} a}{1+\frac{F_{1 y}}{F_{1 x}} a}
$$


We denote $Z_{3}=z, Z_{4}=w$ and estimate

$$
\frac{\frac{F_{2 x}}{F_{1 x}}(z)+\frac{F_{2 y}}{F_{1 x}}(z) a(z)}{1+\frac{F_{1 y}}{F_{1 x}}(z) a(z)}-\frac{\frac{F_{2 x}}{F_{1 x}}(w)+\frac{F_{2 y}}{F_{1 x}}(w) a(w)}{1+\frac{F_{1 y}}{F_{1 x}}(w) a(w)}
$$

After cross multiplying we get denominator bounded away from 0 . Therefore it is enough to estimate two terms

$$
\frac{F_{2 x}}{F_{1 x}}(w)\left(1+\frac{F_{1 y}}{F_{1 x}}(z) a(z)\right)-\frac{F_{2 x}}{F_{1 x}}(z)\left(1+\frac{F_{1 y}}{F_{1 x}}(w) a(w)\right)
$$

and

$$
\frac{F_{2 y}}{F_{1 x}}(w) a(w)\left(1+\frac{F_{1 y}}{F_{1 x}}(z) a(z)\right)-\frac{F_{2 y}}{F_{1 x}}(z) a(z)\left(1+\frac{F_{1 y}}{F_{1 x}}(w) a(w)\right)
$$

Both expressions are estimated similarly. To estimate 35 we split it into

$$
\frac{F_{2 y}}{F_{1 x}}(w) a(w)-\frac{F_{2 y}}{F_{1 x}}(z) a(z)
$$

and

$$
a(z) a(w)\left(\frac{F_{2 y}}{F_{1 x}}(w) \frac{F_{1 y}}{F_{1 x}}(z)-\frac{F_{2 y}}{F_{1 x}}(z) \frac{F_{1 y}}{F_{1 x}}(w)\right)
$$

As above we use elementary algebra and get expressions of the type

$$
\frac{F_{1 x}(w)-F_{1 x}(z)}{F_{1 x}(z)}
$$

and

$$
\frac{F_{2 y}(w)-F_{2 y}(z)}{F_{1 x}(z)}
$$

We split $\gamma_{3}$ into small intervals, and apply the mean value theorem. The ratios $\frac{F_{1 x}(\theta)}{F_{1 x}(z)}$ or equivalently the differences $\log F_{1 x}(\theta)-\log F_{1 x}(z)$ for close points $\theta, z$ on the same stable manifold are estimated (using again the mean value theorem and D2) as

$$
\log F_{1 x}(\theta)-\log F_{1 x}(z)<C_{0}(1+\alpha) \Delta y
$$

Thus for any two points $z$ and $\theta$ on the same stable manifold

$$
\log F_{1 x}(\theta)-\log F_{1 x}(z)<C|z-\theta|
$$


In particular for all points $z$ and $\theta$ on the same stable manifold the ratios $\frac{F_{1 x}(\theta)}{F_{1 x}(z)}$ are uniformly bounded.

Thus estimate 37 contributes

$$
C\left|\gamma_{3}\right|
$$

When estimating 36 we get similar terms estimated as 42 , and

$$
\frac{F_{2 y}}{F_{1 x}}(z)(a(z)-a(w))
$$

After we combine all terms except 43 we get an estimate

$$
M_{0} C_{0} \frac{1}{K_{0}^{m}}
$$

where $M_{0}$ is a uniform constant, which depends on the number of similar terms that we added above, and $C_{0}$ is the distortion constant from condition D2. For 43 we use inductive assumption 30 and get a total estimate

$$
\left|a_{F\left(Z_{3}\right)}-a_{F\left(Z_{4}\right)}\right|<M_{0} C_{0} \frac{1}{K_{0}^{m}}+\left(\frac{1}{K_{0}^{2}}+\alpha^{2}\right) c_{0} \theta_{0}^{m}
$$

As $K_{0}>1$ we can choose $\theta_{0}<1$ satisfying for some $A_{0}>1$

$$
\theta_{0}=\frac{A_{0}}{K_{0}}
$$

Also H5 implies that we can choose $\theta_{0}<1$ satisfying simulteneously

$$
\frac{1}{K_{0}^{2}}+\alpha^{2}<\theta_{0}
$$

Then if

$$
c_{0}>\frac{M_{0} C_{0}}{\theta_{0}-\frac{1}{K_{0}^{2}}+\alpha^{2}}
$$

we get the left side of 45 less than $c_{0} \theta_{0}^{m+1}$.

Q.E.D.

From Lemma 5.2 and 29 we get

$$
\left|\log D^{u} F\left(z_{3}\right)-\log D^{u} F\left(z_{4}\right)\right|<C_{3} \theta_{0}^{m}
$$

Combining 26, 27, 49 we conclude the proof of Proposition 5.1. 
2. We combine several corollaries from Proposition 5.1 and from the arguments used in its proof.

Corollary 5.3 There exists $c$ independent of $i$ such that for any $z_{1}, z_{2} \in E_{i}$ holds

$$
\frac{\left|f_{i 1 x}\left(z_{1}\right)\right|}{\left|f_{i 1 x}\left(z_{2}\right)\right|}<c
$$

Here $z_{1}, z_{2}$ do not need to be on the attractor.

To prove Corollary 5.3 we fix an arbitrary stable manifold $W_{i}^{s} \subset E_{i}$, and connect $z_{1}$ to $z_{3} \in W_{i}^{s}$ by a horizontal segment $\sigma$. As full height rectangles are bounded from above and below by horizontal segments, $\sigma$ lies entirely in $E_{i}$. Similarly we connect $z_{2}$ to $z_{4} \in W_{i}^{S}$. Then 22 and 41 imply 50 . Let $\delta_{z}\left(E_{i}\right)$ be the width of the horizontal crossection of $E_{i}$ through $z \in E_{i} \cap \Lambda$. As $\delta_{z}\left(E_{i}\right)$ are mapped onto full width unstable curves we get from 50

Corollary 5.4 There exists $c$ independent of $i$ such that for any $z_{1}, z_{2} \in E_{i}$ holds

$$
\frac{\delta_{z_{1}}\left(E_{i}\right)}{\delta_{z_{2}}\left(E_{i}\right)}<c
$$

Remark 5.5 Property 51 demonstrates restrictions on geometry imposed by condition $D 2$.

Conditions of Theorem 3.1 allow widths of $E_{i}$ to oscillate exponentially between $a^{i}$ and $b^{i}$ for some $0<a<b<1$. However from 51 we get that ratios are uniformly bounded.

Applying 9 to the full height rectangles $E_{i}$ we get for all $z_{1}, z_{2} \in E_{i} \cap \Lambda$

\section{Corollary 5.6}

$$
\operatorname{var}\left(\log D^{u} F\right) \mid E_{i}<C
$$

and

\section{Corollary 5.7}

$$
\frac{D^{u} f_{i}\left(z_{1}\right)}{D^{u} f_{i}\left(z_{2}\right)}<c
$$


For any $z$ on an unstable curve $W^{u}(z) \subset E_{i}$ which is full width in $E_{i}$ let $\left|W^{u}\left(z, E_{i}\right)\right|$ be the respective length . As $\left|W^{u}\left(z, E_{i}\right)\right|$ coincide up to a uniformly bounded factor with $\frac{1}{D^{u} f_{i}(z)}$ we get from 53

Corollary 5.8 There exists $c$ independent of $i$ such that for any $z_{1}, z_{2} \in E_{i} \cap$ $\Lambda$ holds

$$
\frac{\left|W^{u}\left(z_{1}, E_{i}\right)\right|}{\left|W^{u}\left(z_{2}, E_{i}\right)\right|}<c
$$

Note that 54 also follows from 51 because at a given point $z \in E_{i}$ ratios : $\frac{\delta_{z}\left(E_{i}\right)}{\left|W^{u}\left(z_{1}, E_{i}\right)\right|}$ are uniformly bounded.

Although the next statement is not used in the proof of the main theorem , it is usefull for understanding the geometry of partitions into $E_{i_{0} \ldots i_{n-1}}$. We claim that 54 is valid for rectangles of any order.

Remark 5.9 Let $E_{i_{0} \ldots i_{n-1}}$ be a full height rectangle of order $n$.

Then for any two points $z_{1}, z_{2} \in E_{i_{0} \ldots i_{n-1}} \cap \Lambda$ holds

$$
\frac{\left|W^{u}\left(z_{1}, E_{i_{0} \ldots i_{n-1}}\right)\right|}{\left|W^{u}\left(z_{2}, E_{i_{0} \ldots i_{n-1}}\right)\right|}<c
$$

To prove 55 we rewrite the ratio $\frac{D^{u} F^{n}\left(z_{2}\right)}{D^{u} F^{n}\left(z_{1}\right)}$ as

$$
\frac{\prod_{i=0}^{n-1} D^{u} F\left(F^{i} z_{2}\right)}{\prod_{i=0}^{n-1} D^{u} F\left(F^{i} z_{1}\right)}
$$

and consider

$$
\sum_{i=0}^{n-1}\left|\log D^{u} F\left(F^{i} z_{2}\right)-\log D^{u} F\left(F^{i} z_{1}\right)\right|
$$

As in the proof of Proposition 5.1 we split the estimate of each term into estimates along stable and unstable manifolds in the images $F^{p}\left(E_{i_{0} \ldots i_{n-1}}\right)=$ $R_{i_{0} \ldots i_{p-1}, i_{p} \ldots i_{n-1}}$. For each term estimates from the proof of Proposition 5.1 imply respective bounds : $C \theta_{0}^{p}$ on stable manifolds, and $C \frac{1}{K_{0}^{n-p}}$ on unstable manifolds. Thus we get a uniform bound in 57, which implies 55. 
3. According to 2.3 points of $\Lambda$ are identified with two-sided sequences

$$
\left(\ldots i_{-m} \ldots i_{-1}, i_{0} i_{1} \ldots i_{n} \ldots\right)
$$

In order to use Ruelle-Bowen approach we define a function $\phi^{u}$ corresponding to $-\log D^{u} F(z)$ on the space of one-sided sequences. We fix some unstable manifold $W_{0}^{u}$. Let $z=(x, y) \in \Lambda, z_{0}=W^{s}(z) \cap W_{0}^{u}$. For $\phi(z)=-\log D^{u}(z)$ define

$$
u(z)=\sum_{k=0}^{\infty} \phi\left(F^{k}(z)\right)-\phi\left(F^{k}\left(z_{0}\right)\right)
$$

and

$$
\psi(z)=\phi(z)-u(z)+u(F z)
$$

From 9 we get that the series 58 converge uniformly.

For $\psi(z)$ all terms with $z$ cancel, so $\psi(z)$ as a function of $z_{0}$ depends only on nonnegative iterates of $z$.

Let $\Omega_{+}=\left\{x=\left(i_{0} i_{1} \ldots i_{n} \ldots\right)\right\}$ be the space of one-sided sequences corresponding to stable manifolds.

The above function $\psi(z)$ defined on $\Omega_{+}$will be denoted $\phi^{u}(x)$. On the space $\Omega_{+}$we use the metric $d\left(x^{1}, x^{2}\right)=2^{-n}$, where $n=\min \left\{k: i_{k}^{1} \neq i_{k}^{2}\right\}$.

For a function $\phi(x)$ on the symbolic space $\Omega_{+}$let

$$
V_{n}(\phi)=\sup |\phi(x)-\phi(y)|: x_{i}=y_{i}, i=0, \ldots, n-1
$$

$\phi(x)$ is called locally Hölder if there are $C>0,0<\theta<1$ such that $\forall n \geq 1$

$$
V_{n}(\phi)<C \theta^{n}
$$

We use the same arguments as in [6] which prove that the Hölder property on the space of two-sided sequences implies the Hölder property for respective function on the space of one-sided sequences. Then Proposition 5.1 implies

Corollary $5.10 \phi^{u}(x)$ is a locally Hölder function on the symbolic space $\Omega_{+}$. 


\section{Some sufficient conditions for exponential decay of correlations in countable shifts}

1. We refer to [12] for the following general results about shifts with countable alphabets .

Let $T$ be the shift transformation on the space $X$ of admissible one-sided sequences determined by an infinite matrix $A$ with $a_{i j}=0,1$. Here $i, j$ are states of an infinite alphabet. Let $C=\left[i_{0}, \ldots, i_{n-1}\right]$ be cylinder sets. The system $(X, A, T)$ is called topologically mixing if the following holds.

$$
\forall C_{1}, C_{2} \quad \exists N\left(C_{1}, C_{2}\right): \forall n>N\left(C_{1}, C_{2}\right) C_{1} \cap T^{-n} C_{2} \neq \emptyset
$$

for any two cylinder sets $C_{1}$ and $C_{2}$.

Let $\left(X_{A}, T\right)$ be a topologically mixing countable shift, and let $\phi(x)$ be a locally Hölder function.

Set $\phi_{n}(x)=\sum_{k=0}^{n-1} \phi \circ T^{k}(x)$. Define $Z_{n}(\phi, a)$ by

$$
Z_{n}(\phi, a)=\sum_{T^{n} x=x, x_{0}=a} e^{\phi_{n}(x)}
$$

Then

$$
P(\phi)=\lim _{n \rightarrow \infty} \frac{1}{n} \log Z_{n}(\phi, a)
$$

does not depend of the choice of $a$.

Definition Assuming $\left(X_{A}, \sigma\right)$ is topologically mixing and $\phi$ is locally Hölder continuous $\phi$ is called positive recurrent if there is $\lambda>0$ such that for any given symbol $a$ there is a constant $M_{a}>1$ and an integer $N_{a}$ such that for every $n \geq N_{a}$ holds

$$
\frac{Z_{n}(\phi, a)}{\lambda^{n}} \in\left[M_{a}^{-1}, M_{a}\right]
$$

Let $L_{\phi}$ be the Ruelle operator

$$
L_{\phi} f(x)=\sum_{T y=x} e^{\phi(y)} f(y)
$$

The following is a part of Theorem 4 in [12]. 
Theorem 6.1 Let $\left(X_{A}, T\right)$ be a topologically mixing countable shift, and let $\phi(x)$ be a locally Hölder function such that $P(\phi)<\infty$. If $\phi$ is positive recurrent, then $\lambda=e^{P(\phi)}$ and there exist $a \sigma$-finite measure $v$ and a function $h>0$ such that $L_{\phi}^{*} v=\lambda v, L_{\phi} h=\lambda h, \quad v(h)=1$, and for every uniformly continuous function $f$ such that $\left\|f h^{-1}\right\|_{\infty}<\infty$ holds

$$
\lambda^{-n} L_{\phi}^{n} f \rightarrow v(f) h
$$

uniformly on compacts.

Consider the space of functions $\mathscr{L}$ with bounded norm $|f|_{\mathscr{L}}$ which is the sum of $\|f\|_{\infty}$ and some fixed Hölder norm. The next theorem which follows from several results in [12] implies exponential decay of correlations for Hölder functions restricted to the attractor.

Theorem 6.2 Suppose the following properties are satisfied.

a) $\left(X_{A}, T\right)$ is topologically mixing.

b) $P(\phi)<\infty$.

c) $\phi$ is positive recurrent.

d) $h$ is bounded away from 0 and $\infty$.

Then there exist $K>0, \theta \in(0,1)$ such that for $f \in \mathscr{L}$ holds

$$
\left|\lambda^{-n} L_{\phi}^{n} f-h v(f)\right|_{\mathscr{L}}<K \theta^{n}|f|_{\mathscr{L}}
$$

In general for infinite shifts none of the properties a),b),c),d) is automatic. The following property was introduced by Aaronson,Denker,Urbanski ([2] $)$ and Yuri ([16]).

\section{Finitely Many Images Property .}

The number of different rows of the matrix $A$ is finite.

In the case of finitely many images the following results from [12] simplify the set of properties sufficient for exponential decay of correlations.

(i) If $A$ satisfies finitely many images property and $\left|L_{\phi}(1)\right|_{\infty}<\infty$, then $\phi$ is positive recurrent .

(ii) If $\phi$ is positive recurrent and $P(\phi)<\infty$, then $h$ is bounded away from 0 and infinity. 
2. As a corollary from the above results from [12] : theorems 6.1, 6.2, and properties (i) and (ii) we get

Proposition 6.3 Suppose there is a Markov partition of the attractor satisfying the following properties.

(a) The matrix A of admissible transitions is topologically mixing and has finitely many different rows.

(b) $\Phi(x, y)=-\log \left|D^{u} F\right|$ is Hölder on the space of admissible sequences.

(c) For some $\phi(x)$ cohomologous to $\Phi(x, y)$ holds $P(\phi(x))<\infty$.

(d) Norm $\left|L_{\phi}(1)\right|_{\infty}$ is finite.

then 65 holds.

Proposition 6.3 gives sufficient conditions for exponential decay of correlations for Hölder (in particular smooth) functions restricted to the attractor.

\section{Proof of the exponential decay of correlations}

We check properties $(a)-(d)$.

1. Recall that in our model we consider the partition of the square into full height rectangles $E_{i}$.

Our shift is Bernoulli, all rows (and columns) are the same row of 1-s, so it is topologically mixing and property $(a)$ is satisfied.

2. Property $(b)$ follows from 9 .

3. Next we prove property $(c)$. As in the case of attractors for Axiom A systems we prove

Proposition 7.1 For $\phi(x)=\phi^{u}(x)$ topological pressure $P\left(\phi^{u}(x)\right)$ equals zero.

\section{Proof.}

We fix some symbol $a$, respective rectangle $E_{a}$, and $W_{0 a}^{u}=W_{0}^{u} \cap E_{a}$. When evaluating $Z_{n}(\phi, a)$ in 62 we consider respective sum over all periodic orbits of period $n$ starting in $E_{a}$. 
Each cylinder set $E_{a i_{1} \ldots i_{n-1}}$ contains one periodic orbit of period $n$. When evaluating $\phi^{u}(x)$ we use formula 59. We can evaluate that expression at a point $z$ of intersection between the stable manifold of a periodic point in $E_{a i_{1} \ldots i_{n-1}}$ and $W_{0 a}^{u}$. Then each term in 62 is a product of two expressions.

The first expression equals $\frac{1}{D^{u} F^{n}(z)}$, which coincides up to a uniformly bounded factor with the length of $W^{u}\left(z, E_{a i_{1} \ldots i_{n-1}}\right)$.

The second expression $e^{u(z)-u(F(z)}$ is uniformly bounded away from zero and infinity.

Therefore up to a uniformly bounded factor the sum 62 equals to the length of $W_{0 a}^{u}$. That implies $P(\phi)=0$. Q.E.D.

4. Property $(d)$ is an easier version of the above proposition. For any $x$ the expression $L_{\phi}(1(x))$ equals up to a uniform constant

$$
\sum_{i}\left|W_{0}^{u}\left(x_{i}, E_{i}\right)\right|
$$

where $x_{i} \in E_{i}$ is the point of intersection between the stable manifold of the respective preimage of $x$ and $W_{0}^{u}$. Therefore $\left|L_{\phi}(1)\right|_{\infty}<\infty$ is bounded by $c\left|W_{0}^{u}\right|$.

So all properties of Proposition 6.3 are satisfied, and we get exponential decay of correlations for one-sided shift. As in [6] it implies exponential decay of correlations for two-sided shift and therefore for Hölder functions on $Q$. That proves Theorem 4.2 .

Remark 7.2 65also implies the central limit theorem for for Hölder functions on $Q$.

Let us denote $\mu_{S}$ the invariant measure on $\Lambda$ constructed in [8], [9] following Sinai method, and let $\mu_{R B}$ be the invariant measure on $\Lambda$ constructed above following Ruelle-Bowen method, see [11], [6]. Let $\mu_{1}$ be the projection of $\mu_{S}$ onto one-sided sequences, and let $\mu$ be the measure on one-sided sequences constructed above by Ruelle-Bowen method. In both constructions measures of cylinder sets $\left[i_{0} i_{1} \ldots i_{n-1}\right]$ of any rank equal up to a uniform constant to the length of the crossections of $E_{i_{0} \ldots i_{n-1}}$ by $W_{0}^{u}$. So $\mu_{1}$ and $\mu$ are equivalent and therefore they coincide. That is a particular case of the characterization of Gibbs measures proved in [12].

As in the classical case that implies 
Corollary 7.3 Measures $\mu_{S}$ and $\mu_{R B}$ coincide.

Acknowledgements. I want to thank Sheldon Newhouse, David Ruelle and Omri Sarig for useful discussions during the preparation of this paper.

\section{References}

[1] J. Aaronson and M. Denker. Ergodic Local limit theorems for Gibbs-Markov maps. Preprint, 1996.

[2] J. Aaronson, M. Denker, M. Urbanski Ergodic Theory for Markov fibered systems and parabolic rational maps. Trans. AMS , 337, 495-548, 1993.

[3] R. Adler. Afterword to R. Bowen, Invariant measures for Markov maps of the interval. Comm. Math. Phys., 69(1):1-17, 1979.

[4] V. M. Alekseev. Quasi-random dynamical systems, I. Math. of the USSR, Sbornik, 5(1):73-128, 1968.

[5] D. V. Anosov and Ya. G. Sinai. Some smooth ergodic systems. Russian Math. Surveys, 22: 103-167, 1967.

[6] R.Bowen. Equilibrium states and the ergodic theory of Anosov diffeomorphisms. Lecture Notes Math., 470, Springer-Verlag, 1975.

[7] M. Hirsch and C. Pugh. Stable manifolds and hyperbolic sets. Proc. AMS Symp. Pure Math., 14, 1970.

[8] M. V. Jakobson and S. E. Newhouse. A two dimensional version of the folklore theorem. American Math. Soc. Translations, Series 2, 171:89-105, 1996.

[9] M. V. Jakobson and S. E. Newhouse. Asymptotic measures for hyperbolic piecewise smooth mappings of a rectangle. Astérisque, 261: 103-160, 2000.

[10] C. Pugh and M. Shub. Ergodic attractors. Transactions AMS, 312(1):1-54, 1989.

[11] David Ruelle. A measure associated with axiom A attractors. Amer. J. Math. 98, n. 3, 619-654, 1976. 
[12] O. Sarig. Thermodynamic formalism for countable Markov shifts. Ergodic Theory Dynam. Systems, 19, n. 6 : 1565-1593, 1999.

[13] Ya.G. Sinai. Topics in Ergodic Theory. Number 44 in Princeton Mathematical Series. Princeton University Press, 1994.

[14] Stephen Smale. Diffeomorphisms with many periodic points. Differential and combinatorial Topology ( A Symposium in Honor of Marstone Morse), Princeton University Press, 1965, 63-80.

[15] Peter Walters. Invariant measures and equilibrium states for some mappings which expand distances. Trans. AMS, 236:121-153, 1978.

[16] M. Yuri. Multi-dimensional maps with infinite invariant measures and countable state sofic shifts. Indag.Math, 6, 355-383, 1995. 